Subscriber access provided by CORNELL UNIVERSITY LIBRARY

\title{
Article
}

\section{Dynamics of Dissolutive Wetting: A Molecular Dynamics Study}

Quanzi Yuan, Jinhong Yang, Yi Sui, and Yapu Zhao

Langmuir, Just Accepted Manuscript • DOI: 10.1021/acs.langmuir.7b01154 • Publication Date (Web): 08 Jun 2017

Downloaded from http://pubs.acs.org on June 15, 2017

\section{Just Accepted}

"Just Accepted" manuscripts have been peer-reviewed and accepted for publication. They are posted online prior to technical editing, formatting for publication and author proofing. The American Chemical Society provides "Just Accepted" as a free service to the research community to expedite the dissemination of scientific material as soon as possible after acceptance. "Just Accepted" manuscripts appear in full in PDF format accompanied by an HTML abstract. "Just Accepted" manuscripts have been fully peer reviewed, but should not be considered the official version of record. They are accessible to all readers and citable by the Digital Object Identifier (DOI®). "Just Accepted" is an optional service offered to authors. Therefore, the "Just Accepted" Web site may not include all articles that will be published in the journal. After a manuscript is technically edited and formatted, it will be removed from the "Just Accepted" Web site and published as an ASAP article. Note that technical editing may introduce minor changes to the manuscript text and/or graphics which could affect content, and all legal disclaimers and ethical guidelines that apply to the journal pertain. ACS cannot be held responsible for errors or consequences arising from the use of information contained in these "Just Accepted" manuscripts. 


\title{
Dynamics of Dissolutive Wetting: A Molecular
}

\section{Dynamics Study}

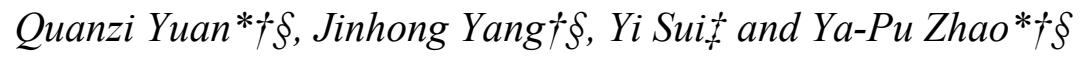 \\ $\uparrow$ State Key Laboratory of Nonlinear Mechanics, Institute of Mechanics, Chinese Academy of \\ Sciences, Beijing 100190, People's Republic of China \\ $\S$ School of Engineering Science, University of Chinese Academy of Sciences, Beijing 100049, \\ People's Republic of China \\ $\$$ School of Engineering and Materials Sciences, Queen Mary University of London, Mile End \\ Road, London E1 4NS, United Kingdom
}

\section{AUTHOR INFORMATION \\ Corresponding Authors \\ *E-mail: yzhao@imech.ac.cn and yuanquanzi@lnm.imech.ac.cn}

\begin{abstract}
Author Contributions
The manuscript was written through contributions of all authors. All authors have given approval to the final version of the manuscript.
\end{abstract}




\begin{abstract}
Dissolutive wetting, i.e. dynamic wetting of a liquid droplet on dissolvable substrates, has been studied by molecular dynamics simulations. In dissolutive wetting, geometry and properties of the solid-liquid interface evolve with the solid dissolving into the droplet, meanwhile the droplet spreads on the receding solid surfaces. The droplets advance on dissolvable substrate following different dynamic laws, compared with spreading on non-dissolutive substrate. Based on molecular kinetic theory, we develop a theoretical model to reveal physical mechanisms behind the dissolutive wetting phenomena. We also find that solid particles are pulled by their hydration shells to dissolve into liquid, changing the flow field, the atomic structure and the hydrogen bond network in the droplet. Our findings may help to comprehend the dynamics of dissolutive wetting and assist future design in practical applications.
\end{abstract}

\title{
KEYWORDS
}

Wetting, Dissolution, Solid-liquid interface, Molecular dynamics 


\section{INTRODUCTION}

Dissolutive wetting, i.e. dynamic wetting of liquids on dissolvable solid surfaces, is a subject of significant interest in a broad class of natural and industrial processes, such as weathering of materials, ${ }^{1-2}$ alloy formation, ${ }^{3-5}$ erosion, ${ }^{6-8}$ drug release, ${ }^{9-11}$ biomedicine, ${ }^{12-13}$ self-propelling, ${ }^{14-16}$ amongst other. ${ }^{17-18}$ To utilize dissolutive wetting in practical applications, the researchers have made efforts to explore and comprehend the dynamic dissolving behaviors. ${ }^{3,12,19-21}$ Recently, the very interesting works of utilizing the combination of "dissolvable wetting" and "coffee ring" to form surface structures have been approached experimentally. ${ }^{22-23}$ However, owing to the complexity of the coupling between the dissolution and the fluid flow, dynamics of dissolutive wetting is still far from being well understood.

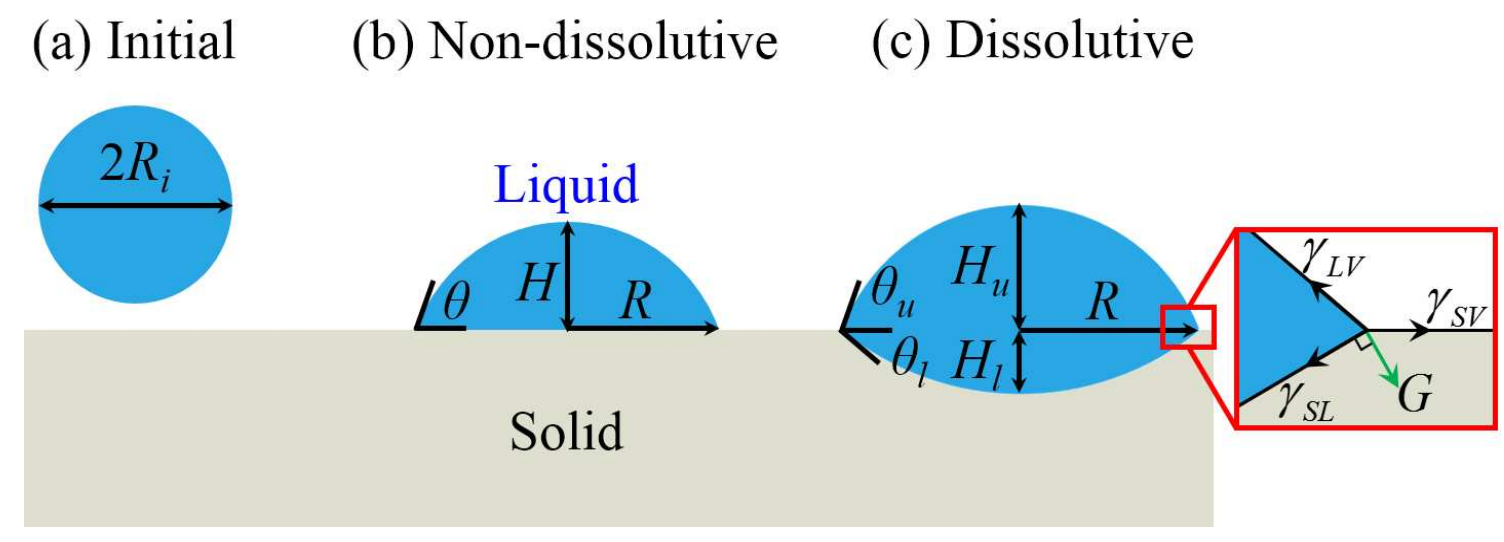

Figure 1. Illustrations of (a) the initial state of a droplet with radius of $R_{i}$ on a solid, (b) nondissolutive wetting, and (c) dissolutive wetting. $R, H$ and $\theta$ are the instant radius, height and contact angle, respectively. The subscript $u$ and $l$ represent upper and lower, respectively. $\gamma_{L V}$, $\gamma_{S V}$ and $\gamma_{S L}$ are the liquid-vapor, solid-vapor and solid-liquid interface energies, respectively. $G$ is the dissolving energy, which is perpendicular to the solid-liquid interface. 
Wetting dynamics of liquids on inert solids has been well studied (Figure 1b). ${ }^{24-25}$ The liquid behaviors are governed by balance between driving energies (interface energies, gravity, electric field, etc.) and energy dissipation (viscous resistance, molecular friction, inertia, etc.) in the vicinity of the triple phase region. ${ }^{26-28}$ Dynamic properties of a wetting system could be characterized by the scaling exponent $n$, with spreading radius $R \propto t^{n}$, where $t$ is the spreading time. ${ }^{24}$ For examples: when driving force is surface tension and viscous resistance dissipates, $n=1 / 10$, known as Tanner's law; ${ }^{29}$ surface tension versus molecular friction (see Theoretical Analysis - Molecular kinetic theory) leads to $n=1 / 7{ }^{30} n$ varies from 0 to about 0.25 according to the electric field in electrowetting. ${ }^{26}$ However, scaling laws of dissolutive wetting, i.e. the general dynamic properties, are far from well understood.

In dissolutive wetting (Figure 1c), controlled by dissolving and interface energies, ${ }^{31}$ the solid dissolves into the interface liquid and diffuses towards the bulk liquid, changing the liquid and interface properties, i.e. viscosity, interface energies and etc. In the meantime, geometry of the solid-liquid interface evolves and the droplet spreads on the receding solid surface. In order to apply dissolutive wetting in the application fields of energy, health, geology, etc., understandings of dynamics of dissolutive wetting are necessary. ${ }^{11}$

In this article, dynamics of droplets on dissolvable solid surfaces has been studied using molecular dynamics (MD) simulations. Driven by the interface and dissolving energies, liquid with evolving properties advanced on receding solid boundary. Based on molecular kinetic theory (MKT), we analyzed the dynamics and the physical mechanisms in the wetting processes. Then, the details, i.e. hydration, flow field, pair distribution function (PDF), hydrogen bonds and etc., are revealed at the atomic level. Our findings may expand our knowledge of dynamics of dissolutive wetting and assist the future design in practical applications. 


\section{METHODOLOGY}

Large-scale MD simulations are carried out to explore dissolutive wettings using LAMMPS. ${ }^{32}$ The simulation domain is illustrated in Figure 1: a liquid droplet with initial radius $R_{i}$ of $3 \mathrm{~nm}$ is placed on a smooth solid surface in a simulation box of $25 * 25 * 25 \mathrm{~nm}^{3}$. The liquid is modeled as extended simple point charge (SPC/E) water with viscosity $\eta=0.729 \mathrm{mPa} \cdot \mathrm{s}^{33}$ density $\rho=994$ $\mathrm{kg} / \mathrm{m}^{3}$, surface tension $\gamma_{L V}=0.0636 \mathrm{~N} / \mathrm{m}^{34}$ close to those of real water at $300 \mathrm{~K}$ and 1 bar. In the SPC/E water model, the oxygen atoms were modeled as charged Lennard-Jones (LJ) particles $\left(\sigma_{O-O}=0.3166 \mathrm{~nm}, \varepsilon_{O-O}=0.650 \mathrm{~kJ} / \mathrm{mol}, q_{O}=-0.8476 \mathrm{e}\right)$, while the hydrogen atoms were charged but without considering LJ interactions between them $\left(\sigma_{H-H}=0.0 \mathrm{~nm}, \varepsilon_{H-H}=0.0 \mathrm{~kJ} / \mathrm{mol}, q_{H}=\right.$ $0.4238 \mathrm{e}$ ). The total potential energy $E_{i j}$ between two atoms $i$ and $j$ separated by $r_{i j}$ is the sum of LJ potential energy and Coulombic pairwise interaction: $E_{i j}=4 \varepsilon_{i j}\left[\left(\sigma_{\mathrm{X}-\mathrm{Y}} / r_{i j}\right)^{12}-\left(\sigma_{\mathrm{X}-\mathrm{Y}} / r_{i j}\right)^{6}\right]+k_{e} q_{\mathrm{X}} q_{\mathrm{Y}} / r_{i j}$, where $\varepsilon$ is the depth of the LJ potential well, and $\sigma$ the zero-crossing distance for the potential, the subscripts $\mathrm{X}$ and $\mathrm{Y}$ represent the types of atoms. The solid particles are set to be uncharged LJ particles with with $\sigma_{\mathrm{SS}}=0.264 \mathrm{~nm}$ and $\sigma_{\mathrm{SL}}$ $=0.29 \mathrm{~nm}$. The solid-liquid interaction $\varepsilon_{S L}$ is fixed to make the solid hydrophilic to the liquid with an equilibrium contact angle be about $70^{\circ}$. The NVT ensemble (constant number of atoms $N$, volume $V$ and temperature $T$ ) is used. The Nosé-Hoover thermostat with a time-step of 1 fs is employed to regulate the temperature at $300 \mathrm{~K}$. The evaporated water molecules quickly saturate the simulation domain, so the evaporation effect could be ignored. The instant configurations are recorded every $0.1 \mathrm{~ns}$.

If the substrate is non-dissolvable, the droplet spreads on a smooth solid surface (Figure 1b). If the substrate is dissolvable, the liquid spreads and erodes the solid surface, whilst the solid dissolves into the liquid (Figure 1c). The liquid above and below the solid surface are roughly 
two spherical caps. So, we used two arcs with different radius to fit the cross-section of droplet. In this way, geometric parameters could be defined and obtained (figure 1c), which describe the tendency of the change of the interface shape.

Follow the Noyes-Whitney equation, ${ }^{35}$ which describes kinetic law of dissolution, and previous works of de Gennes, ${ }^{36}$ we define a normalized quantity $\phi: \phi=0$ represents dissolution does not happen; $\phi=1$ represents dissolution is saturated. The exponential relaxation towards equilibrium is $d \phi / d t=(1-\phi) / t_{c}$, which leads to $\phi=1-\exp \left(-t / t_{c}\right)$, where the characteristic time $t_{c}=L^{2} / D_{e}, L$ the characteristic length and $D_{e}$ the diffusion coefficient. In our cases, $L=R_{i}$, therefore $t_{c} \sim 5 \mathrm{~ns}$.

For a solid particle at the solid-liquid interface, it feels attractions from both the surrounding liquid molecules and solid particles. If the total attraction force from the liquid is smaller than that from the solid, this solid particle is inert. If the total attraction force from the liquid is larger than that from the solid, this solid particle dissolves into liquid. These attraction forces from the liquid and solid depend on $\varepsilon_{S L}$ and $\varepsilon_{S S}$, respectively. Since the solid-liquid (SL) interaction $\varepsilon_{S L}$ is fixed, dissolubility of the solid is controlled by the solid-solid interaction $\varepsilon=\varepsilon_{S S} / \varepsilon_{S L}$. When $\varepsilon \gg 1$, the solid is non-dissolutive; when $\varepsilon \sim 1$, the solid is dissolutive. The smaller $\varepsilon$ is, the higher the solid dissolubility is.

When changes of temperature $T$ and pressure $P$ could be neglected (just like cases in our simulations), the dissolving energy equals to chemical potential per unit area, i.e. variation of Gibbs free energy per unit area when one solid particle dissolves into the liquid. So, the unit of $G$ is $\mathrm{J} / \mathrm{m}^{\wedge} 2$. 
$\tau=0.4$

$\tau=0.8$

$\tau=1.2$

$\tau=1.6$

$\tau=2.0$

(a) $\varepsilon=0.48$

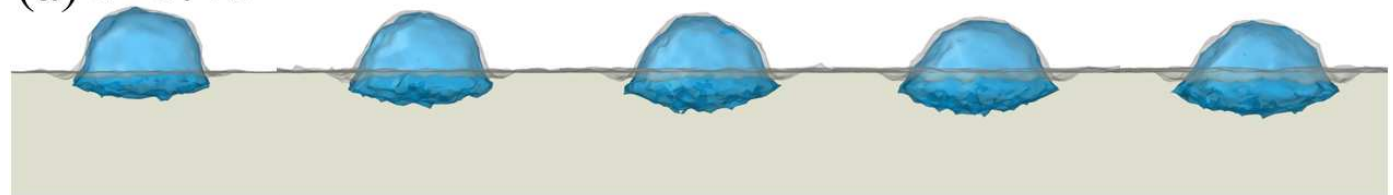

(b) $\varepsilon=0.65$

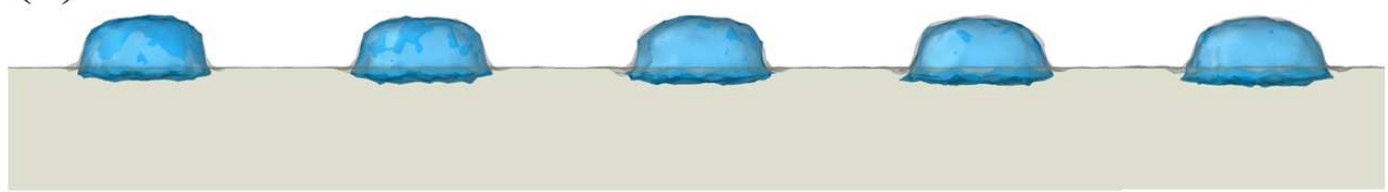

(c) $\varepsilon=0.81$

Liquid

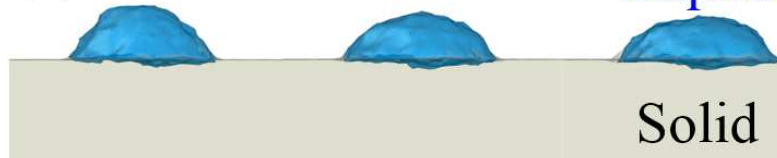

Figure 2. The processes of dissolutive wetting with (a) $\varepsilon=0.48$, (b) $\varepsilon=0.65$ and (c) $\varepsilon=0.81$.

We simulate the wetting processes of the droplet on solid surfaces with different dissolubility. When $\varepsilon=0.81$ (Figure 2c), the solid-liquid interactions slightly overcome the solid-solid interactions, few solid particles dissolve into liquid. When $\varepsilon=0.65$ (Figure 2b), the spreading speed is slower than that in Figure 2c, because part of the driving work is dissipated to erode the solid and to form the lower spherical cap. When $\varepsilon=0.48$ (Figure 2a), the solid-liquid interactions exceed twice of the solid-solid interactions, the solid dissolve in water very fast and liquid sinks into solid. Because each figure in figure 2 is a snapshot of transient moment, fluctuations on the interfaces were caused by thermal fluctuations, which would eliminate when statistically averaged in long period. Then, the geometric parameters are measured and shown in Figures 3a and 4 . The spreading velocity shown in Figure $3 b$ derived from Figure 3a, and the SavitzkyGolay method ${ }^{37}$ has been used to filter the thermal fluctuations. 

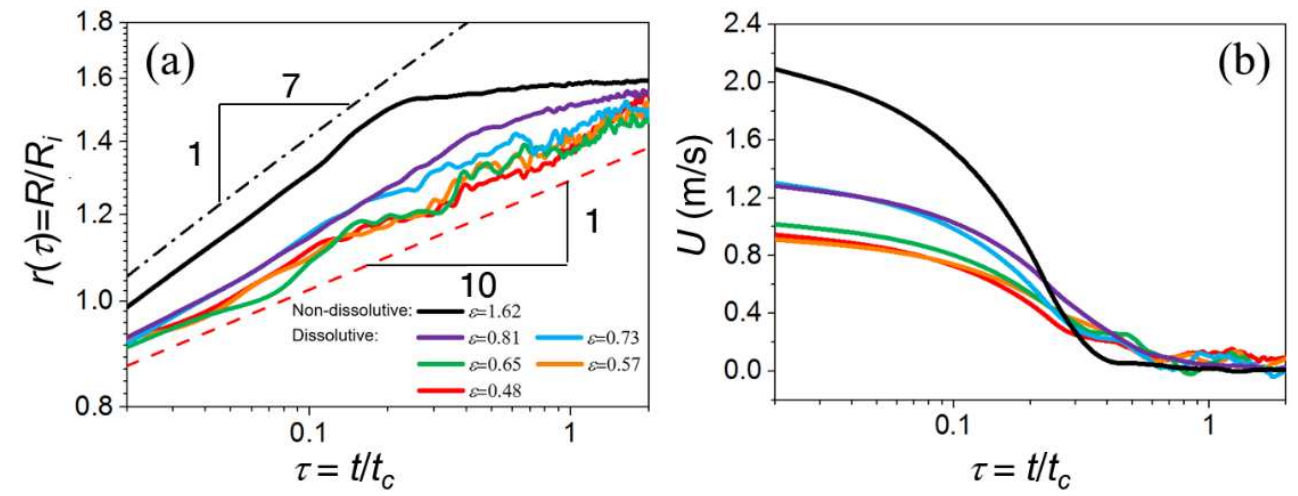

Figure 3. Evolution of (a) radius (log-log plot) and (b) spreading velocity of droplets in MD simulations of wetting on solids with different dissolubility. Black dash-dot and red dashed lines respectively represent scaling laws of $r \sim \tau^{1 / 7}$ and $r \sim \tau^{1 / 10}$ in (a).
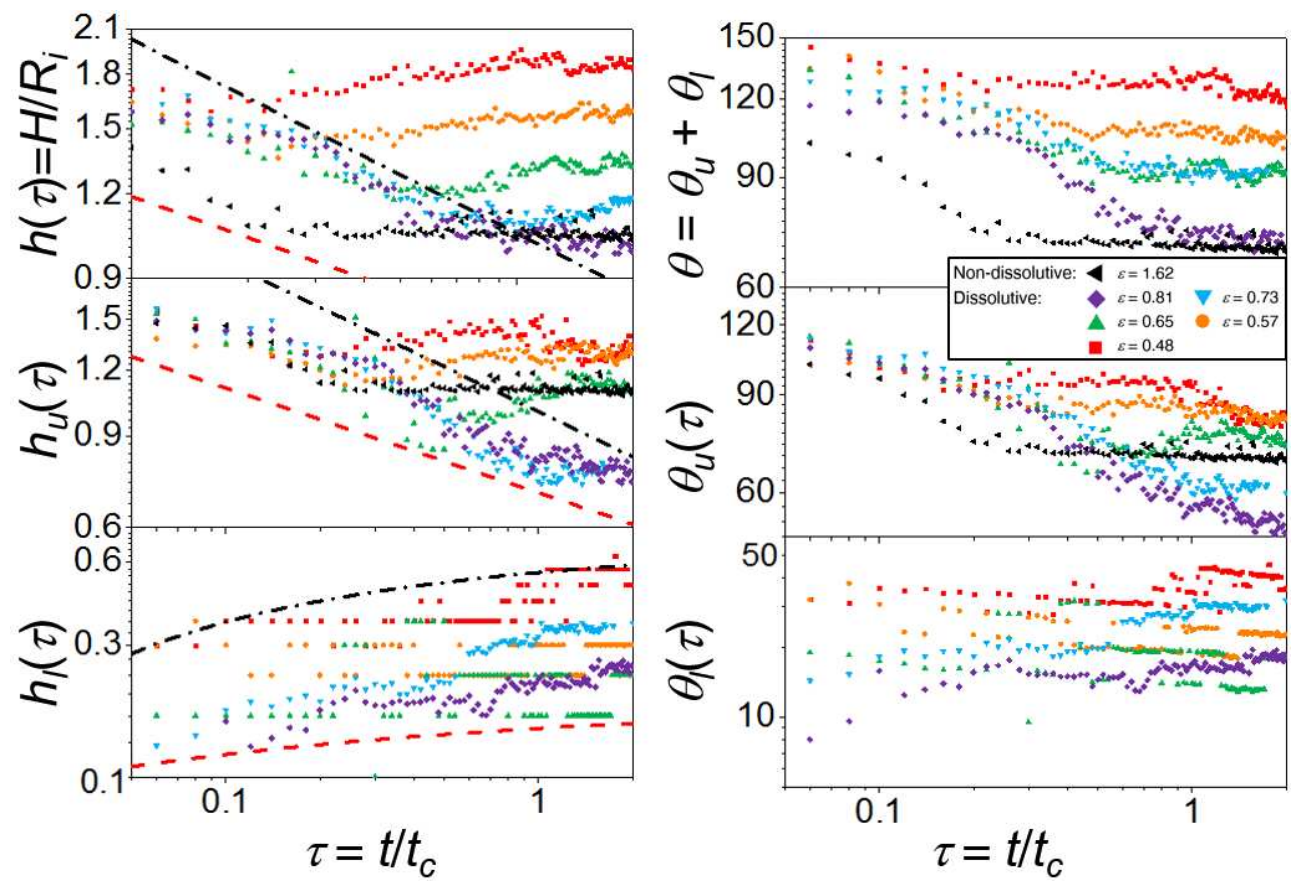

Figure 4. Liquid shape with respect to time in MD simulations (log-log plot). (a) height $h$, (b) upper height $h_{u}$, (c) lower height $h_{l}$, (d) contact angle $\theta$, (e) upper contact angle $\theta_{u}$, and (f) lower contact angle $\theta_{l}$. The black dash-dot and red dashed lines represent scaling laws of $r \sim \tau^{-2 / 7}$ and $r$ $\sim \tau^{-2 / 10}$, respectively. 
Evolutions of radius $r=R / R_{i}$, spreading velocity $U$, height $h=H / R_{i}$, contact angle $\theta$, upper contact angle $\theta_{u}$ and lower contact angle $\theta_{l}$ with respect to time $\tau=t / t_{c}$ for different dissolubility are plotted in Figures 3-4. In non-dissolutive wetting (black line in Figure 3 and black triangles in Figure 4), the liquid spreads with a fast-decayed velocity on the smooth solid surface. Since $\theta_{l}$ is zero, $\theta=\theta_{u}$ decreases fast to its equilibrium value determined by the Young's equation. The scaling law is $r \sim \tau^{1 / 7}$. In dissolutive wetting, the liquid spreads slower than that in nondissolutive wetting (Figure 3b). Further, the spreading velocity decreases with the decrease of $\varepsilon$, because the liquid tends to erode and sink into the solid. Therefore, $r \sim \tau^{\alpha}$, where the scaling exponent $1 / 10<\alpha<1 / 7$ (Figure 3). $h_{u}$ decreases whilst hl increases, resulting in a slower decrease of the droplet height. When $\varepsilon$ is large (purple in Figures 3-4), $h$ and $\theta$ decrease quickly to approach their equilibrium values. When $\varepsilon$ becomes smaller (blue, green, yellow and orange in Figures 3-4), dissolution becomes obvious: $\theta_{u}$ and $h_{u}$ still decrease quickly to approach their equilibrium values, while $\theta_{l}$ and hl gradually increase with speed negatively related to $\varepsilon$. The height of the droplet $h$ first decrease and then slowly increases, and $\theta$ decreases slower than in non-dissolutive wetting, because $\theta_{l}$ and $h_{l}$ gradually increase with speed negatively related to $\varepsilon$. When $\varepsilon$ is small (red line in Figures 3-4), dissolution dominated the process, $h$ first decreases and then slowly increases, while $\theta$ decreases in a different scaling law from the non-dissolutive cases. The dashed and dot lines in Figures 3-4 are eye guides for the power laws of these characteristic quantities of interface shape, which will be discussed in the next part.

\section{THEORETICAL ANALYSIS}

Molecular kinetic theory (MKT). ${ }^{38}$ In Figure 3, the advancing of the contact line obeys the power law of $r \sim \tau^{1 / 7}$ in non-dissolutive wetting, which is validated by previous simulations and 
experiments. ${ }^{30}$ The scaling exponents of the dissolutive spreading range from about $1 / 10$ to $1 / 7$. To uncover the physical mechanisms of dissolutive wetting, we employ MKT to analyze the scaling laws. For a liquid molecule jumps between two adsorption sites separated by a distance $\lambda$ with an equilibrium frequency $\kappa_{0}$, the spreading velocity follows $U=2 \kappa_{0} \lambda \sinh \left(w \lambda^{2} / 2 k_{\mathrm{B}} T\right)$, where $w$ is the driving work, $k_{\mathrm{B}}$ the Boltzmann constant and $T$ the absolute temperature. The adsorption and desorption of liquid molecules on adsorption sites dissipates energy, which is termed as molecular friction $\zeta=k_{\mathrm{B}} T / \kappa_{0} \lambda^{3}$ (unit: $\mathrm{Pa} \cdot \mathrm{s}$ ). ${ }^{38} \kappa_{0}$ is linked to viscosity $\eta$ and the work of adhesion between solid and liquid $W a: \kappa_{0}=\left(k_{\mathrm{B}} T / \eta v_{m}\right) \exp \left(-\lambda^{2} W a / k_{\mathrm{B}} T\right)$, where $v_{m}$ is the molecular flow volume. ${ }^{39}$ In dissolutive wetting, the ratio of driving work $\mathrm{w}$ to thermal energy $k_{\mathrm{B}} T$ is of the order of $0.1 \sim 1, \sinh \left(w \lambda^{2} / 2 k_{\mathrm{B}} T\right) \sim w \lambda^{2} / 2 k_{\mathrm{B}} T$ would be a good approximation to simplify the advancing velocity,

$$
U=\left(\frac{w \lambda^{3}}{\eta v_{m}}\right) \exp \left(-\frac{\lambda^{2} W a}{k_{\mathrm{B}} T}\right) \propto \frac{w}{\eta},
$$

which means spreading velocity is proportional to the driving work, and negative proportional to the viscosity.

Driving work. In dissolutive wetting, a physical quantity $\varphi$, such as the solid-liquid interface energy $\gamma_{S L}$, the liquid-vapor interface energy $\gamma_{L V}$, the dissolving energy $G, \eta$ and etc., evolves in the rule of $\varphi(\tau)=\varphi_{i}+\left(\varphi_{f}-\varphi_{i}\right) \beta(\tau)$, where $\beta(\tau)=1-\exp (-\tau)$ is an aging factor. The subscribe $i$ and $f$ represent the initial and final state, respectively. As shown in Figure 1c, the driving work is

$$
w(\tau)=G(\tau) \sin \theta_{l}(\tau)+\left[\gamma_{L V f} \cos \theta_{u f}-\gamma_{L V}(\tau) \cos \theta_{u}(\tau)\right]+\left[\gamma_{S L f} \cos \theta_{l f}-\gamma_{S L}(\tau) \cos \theta_{l}(\tau)\right] .
$$


Since $\theta_{l}$ increases and $\theta_{u}$ decreases with the time, the $1^{\text {st }}$ (dissolving energy) and the $2^{\text {nd }}$ (liquidvapor interface energy) items promote the advancing of liquid. The $3^{\text {rd }}$ item, which is the variation of the solid-liquid interface energy caused by the geometric change during dissolutive wetting, prevents the advancing of contact line. Therefore, for some liquid-solid pairs, increase of the dissolvability of the solid might enhance the spreading, while increase of the dissolvability of solid might hinder the spreading just like in our simulations. The spreading dynamics is determined by the competition among dissolving, liquid-vapor and solid-liquid interface energies, as well as the evolution of the interface shape.

We adopt the lubrication approximation $\theta \sim H / R \ll 1$. Although it would introduce error into theoretical derivation using the lubrication approximation, this approximation would greatly simplify the derivation and make it easier to see the physical nature of dissolutive wetting. Later on we will show that our simulation results from MD can agree reasonably well with that from the lubrication theory. From Figure 4c, we assume that $\theta_{u}(\tau) \sim a \theta(\tau)$ and $\theta_{l}(\tau) \sim(1-a) \theta(\tau)$ with $a \leq 1$. Because the constant terms do not contribute to the scaling analysis, some of them could be neglected in Equation (2). Therefore,

$$
w(\tau) \propto G(\tau)(1-a) \theta(\tau)+\left[\gamma_{L V}(\tau) a^{2}+\gamma_{S L}(\tau)(1-a)^{2}\right] \theta(\tau)^{2}
$$

Geometric relations. On one hand, the droplet volume $V$ increases with the dissolved particles with volume v: $V(\tau)=V_{i}+v n_{f} \beta(\tau)$, where $n_{f}$ is the final number of dissolved particles, $V_{i}$ the initial volume of the droplet. On the other hand, $V(\tau)=\frac{\pi}{6}\left[3 R(\tau)^{2}+H_{u}(\tau)^{2}\right] H_{u}(\tau)$ $+\frac{\pi}{6}\left[3 R(\tau)^{2}+H_{l}(\tau)^{2}\right] H_{l}(\tau)$. Considering the lubrication approximation, $H_{h}^{2}$ and $H_{l}^{2}$ could be 
neglected compared with $R^{2}$, so $V(\tau) \approx \pi R(\tau)^{2} H(\tau) / 2 \approx \pi R(\tau)^{3} \theta(\tau) / 2$. Therefore, $V(\tau)=V_{i}+v n_{f} \beta(\tau) \approx \pi R(\tau)^{3} \theta(\tau) / 2$

Scaling laws. Substituting geometric, physical and chemical conditions into Equation (1), we obtained the governing equation:

$$
\left\{\begin{array}{c}
\eta(\tau) U(\tau)=\eta(\tau) \dot{R}(\tau) \propto G(\tau)(1-a) \theta(\tau)+\left[\gamma_{L V}(\tau) a^{2}+\gamma_{S L}(\tau)(1-a)^{2}\right] \theta(\tau)^{2} \\
\theta(\tau)=2\left[V_{i}+v n_{f} \beta(\tau)\right] / \pi R(\tau)^{3}
\end{array}\right.
$$

1) In non-dissolutive wetting, i.e. $G=0, \beta(\tau)=1$, and $a=1$, Equation (4) degenerates to the governing equation of non-dissolutive wetting: $\dot{R}(\tau) \propto\left(\gamma_{L V} / \eta\right)\left[R_{i} / R(\tau)\right]^{6}$, which leads to $R / R_{i} \propto \tau^{1 / 7}$ and is validated by previous studies. ${ }^{30}$ Because the volume of the droplet is conserved, $h r^{2} \approx 1, h \approx r^{-2} \propto C_{1}+C_{2} \tau^{-2 / 7}$, the scaling exponent is $-2 / 7$ before $\mathrm{h}$ approaches its equilibrium value, as shown of black dash-dot line in Figures 3-4. Therefore, $\theta \sim h / r \sim C_{1} \tau^{-1 / 7}+C_{2} \tau^{-3 / 7}$, the scaling exponent varies between $-3 / 7$ and $-1 / 7$, and the curve before the equilibrium state can be fitted using the present scaling law.

2) In dissolutive wetting, we vary the physical quantities $\gamma_{L V f} \in\left[\gamma_{L V i}, 2 \gamma_{L V i}\right]$, $\gamma_{S L i} \in\left[\gamma_{L V i}, 5 \gamma_{L V i}\right], \gamma_{S L f} \in\left[\gamma_{S L i}, 2 \gamma_{S L i}\right], \eta_{f} \in\left[\eta_{i}, 2 \eta_{i}\right], G_{i} \in\left[0.1 \gamma_{L V i}, 10 \gamma_{L V i}\right], a \in[0.1,1]$ to take account of possibilities in the studied systems, ${ }^{40-41}$ and numerically solve Equation (4). The scaling law $R / R_{i} \propto \tau^{\alpha}$ has been used to fit the relation between $R$ and $\tau$. We find that the scaling exponent $\alpha$ ranges from 0.085 to 0.147 , which agree with our observations in simulations (Figures 3). Because $h r^{2} \approx 1, h \approx r^{-2} \propto C_{1}+C_{2} \tau^{-2 \alpha}$, the scaling exponent is $2 \alpha$. So, the data of $\mathrm{h}$ before its equilibrium value are between black dash-dot and red 
dashed lines in Figures 4a-c, which represent the scaling laws of $h \propto \tau^{-2 / 7}$ and $h \propto \tau^{-2 / 10}$, respectively. The total contact angle follows $\theta \approx h / r \propto C_{1} \tau^{-\alpha}+C_{2} \tau^{-3 \alpha}$, the scaling exponent varies between $-\alpha$ and $-3 \alpha$, which is also confirmed by our simulations.

3) If the dissolving energy $G$ is much larger than the interface energies, which is not the case in our simulations, Equation (4) could be simplified to the form: $\eta(\tau) \dot{R}(\tau) R(\tau)^{3} \propto G(\tau)\left[V_{i}+v n_{f} \beta(\tau)\right]$. Therefore, $R / R_{i} \propto \tau^{1 / 4}$.

\section{DISCUSSION}

Hydration. Five solid particles are labelled as S1-S5 to show how they interact with the contact line and dissolve in the liquid (Figure 5). When $\tau=0.04$, the droplet gets in contact with the substrate with a fast spreading speed and quickly covers S2, S3 and S4. S4 dissolves into the liquid with a hydration shell, which consisted of about 20 water molecules. S2 and S3 are covered with part of their hydration shells. The hydration shell is composed of the closest water molecules around the dissolved particles, which is labeled by the first peak in the pair distribution function between water molecule and dissolved particle. When $\tau=0.08$, S2 and S3 are pulled by their hydration shells towards the liquid, and the right liquid reaches S5. When $\tau=0.16$, $\mathrm{S} 2$ is pulled into the liquid by its water cage. The liquid advances over S5 and drives it to dissolve. When $\tau=0.32$, S3 and S5 almost dissolve, and the left liquid reaches S1. When $\tau=0.64$, the 5 particles all dissolve into the liquid. Because S2 and S3 are close to each other, their hydration shells attract and get in touch with each other through hydrogen bonds. S1 dissolves into liquid and diffuses along the liquid surface because the spreading velocity at this moment is one order of magnitude slower than that before $\tau=0.08$ (Figure $3 \mathrm{~b}$ ) and the self-diffusion coefficient of the surface liquid is higher than that of the bulk liquid (Figure 6). ${ }^{26}$ So, we can find 
the difference in dissolution of S1 and S5, which is raised from the coupling of dissolution and spreading. When $\tau=1.28$, the water cages of S2 and S3 keep in touch during the simulations. The hydration shells, i.e. flexible water cages composed of about 20 water molecules, play important roles in dissolutive wettings. Particles are pulled by their hydration shell into the water. Then, particle and its hydration shell form a dynamic cluster to interact with other clusters.

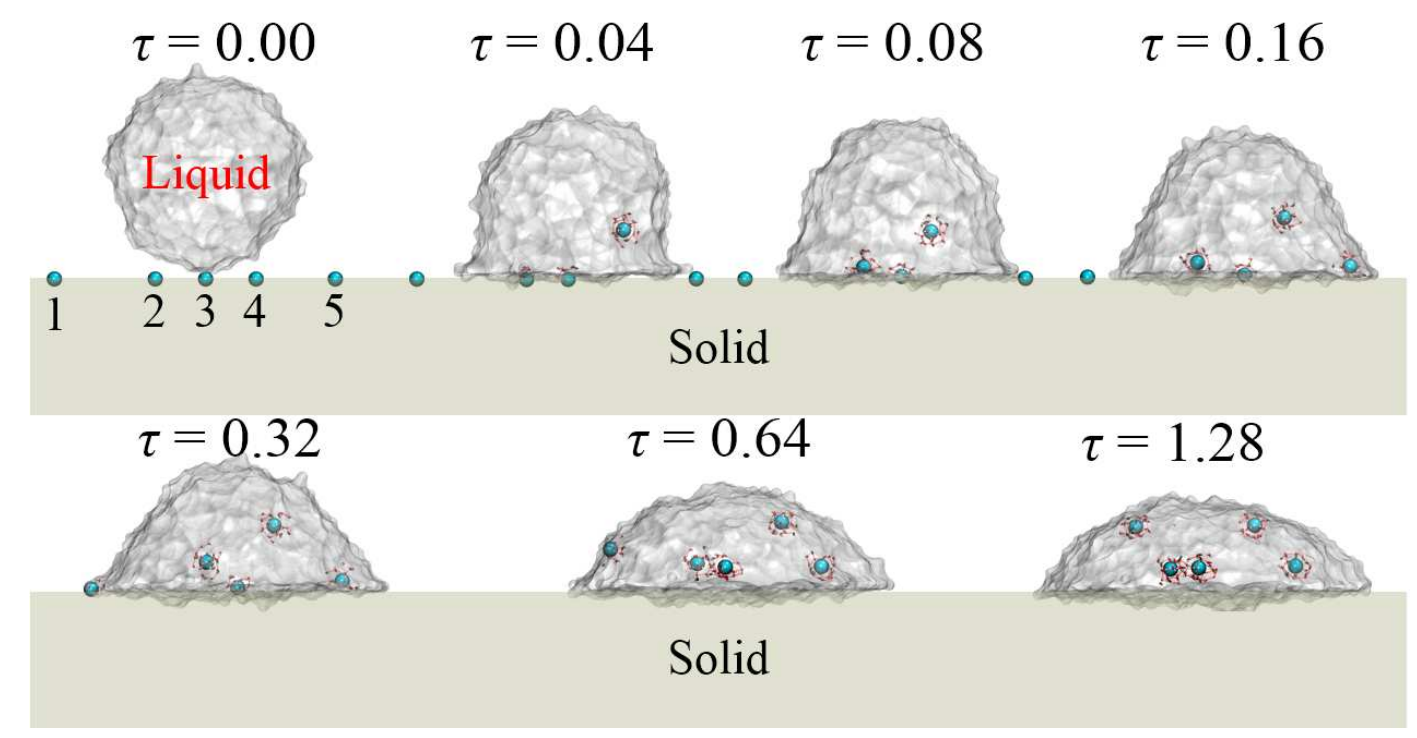

Figure 5. The process of solid particles dissolving in a spreading droplet. The cyan balls represent the solid particles ( $\varepsilon=0.81$ ). The red sticks and lines represent respectively the water molecules and the hydrogen bonds in the hydration shell of the solid particles.

Flow field. When a droplet is deposited on the dissolvable solid, the solid particles began to dissolve into the droplet (Figure 6). The mobility of the water molecules is highest at the droplet surface and lowest at the solid-liquid interface. ${ }^{26}$ Therefore, the dissolving speed of the solid particles is fastest at the triple-phase line and is slowest where solid is immersed in the liquid. ${ }^{42}$ Under the concentration gradient, the dissolved particles diffused along the droplet surface 
towards lower concentration. At the meantime, the water molecules are driven by the surface tension to flow towards the solid and advance along the solid surface to decrease the contact angle.
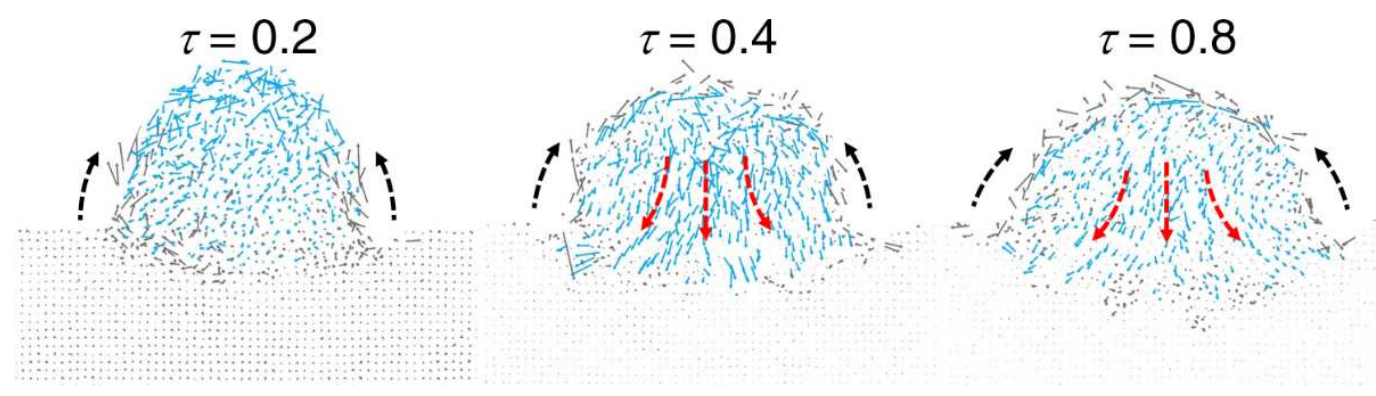

Figure 6. Flow field in dissolutive wetting $(\varepsilon=0.48)$. The blue and gray solid lines show the velocity of water molecules and solid particles, respectively. The length of a line shows the strength of the velocity. The red and black dashed lines are guides for the eyes to show the general tendency.

Pair distribution function (PDF). In non-dissolutive wetting, the structures of both liquid and solid do not change. In dissolutive wetting, the atomic structures of the solvent and solute depend on the solid-liquid interactions. PDF $g(\delta)$ describes the distribution of distances between pairs of molecules between pairs of molecules $\left(\delta=d / \sigma_{S L}\right.$, d: the distance, $\sigma_{S L}$ : the zero-crossing distance between solid and liquid), and is a major descriptor for the atomic structure. In our simulations, the solid has a face-centered-cubic lattice structure and the liquid is water (Figure 7a). Dissolved particles and their hydration shells compose clusters to interact with each other (inset in Figure 7b). Because of the increasing dissolved solids, $g(\delta)$-SS increases with the decrease of $\varepsilon:$ the 1 st peak is heightened, and the 2nd and 3rd peaks became obvious (Figure 
7b). $g(\delta)$-LL decreases with the decrease of $\varepsilon$ (Figure 7c): the PDFs are flattened compared with that of bulk liquid. The first peak in Figure $7 \mathrm{~d}$ labels the position of the nearest water molecules surrounding the solid particles, which composed the hydration shell and is distinct from the bulk water to a distance of about 3 layers.
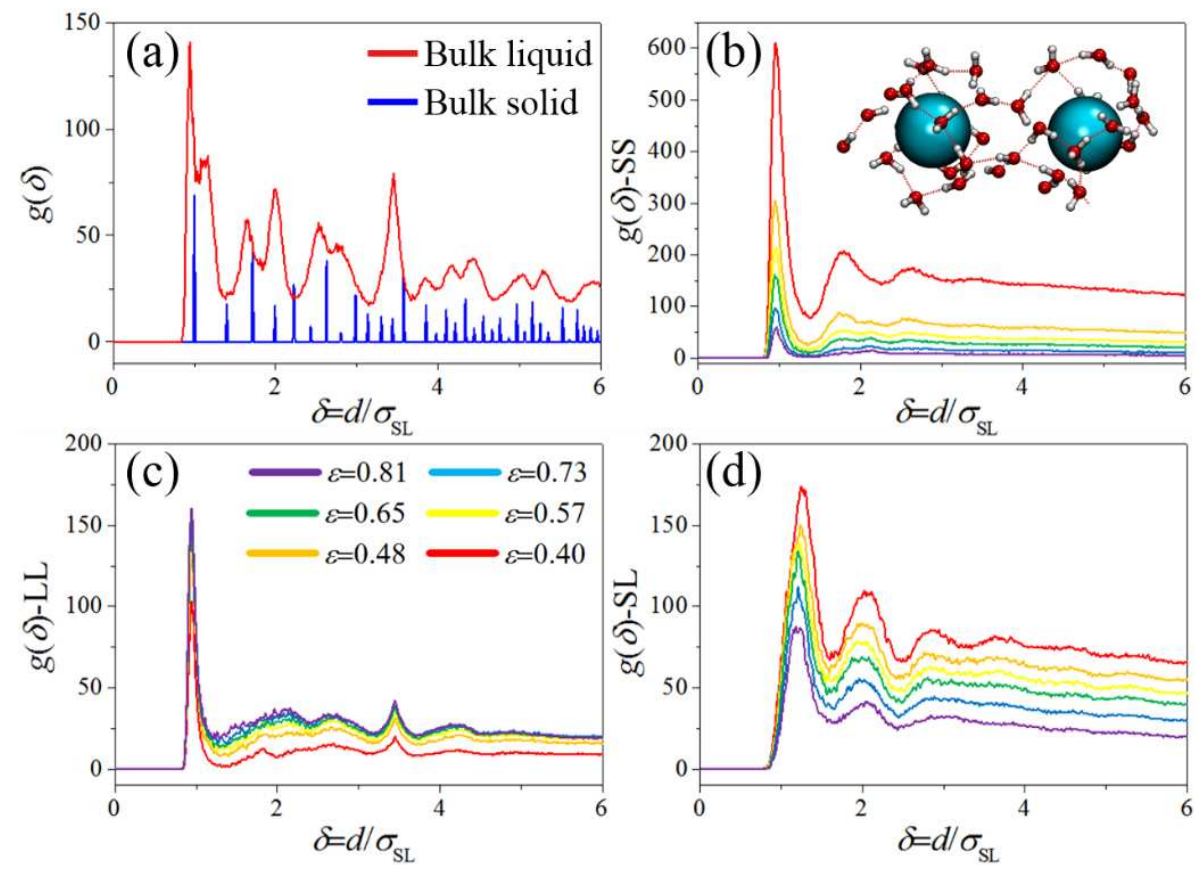

Figure 7. Pair distribution functions $g(\delta)$ (a) between bulk liquid molecules (red) and between bulk solid particles (blue), (b) between dissolved particles, (c) between liquid molecules, and (d) between dissolved solid particles and liquid molecules. The inset in (b) shows two particles interact through their hydration shells. The cyan, red and white balls represent the solid, oxygen and hydrogen atoms, respectively. The red lines represent the hydrogen bonds. 
(a)

(b)

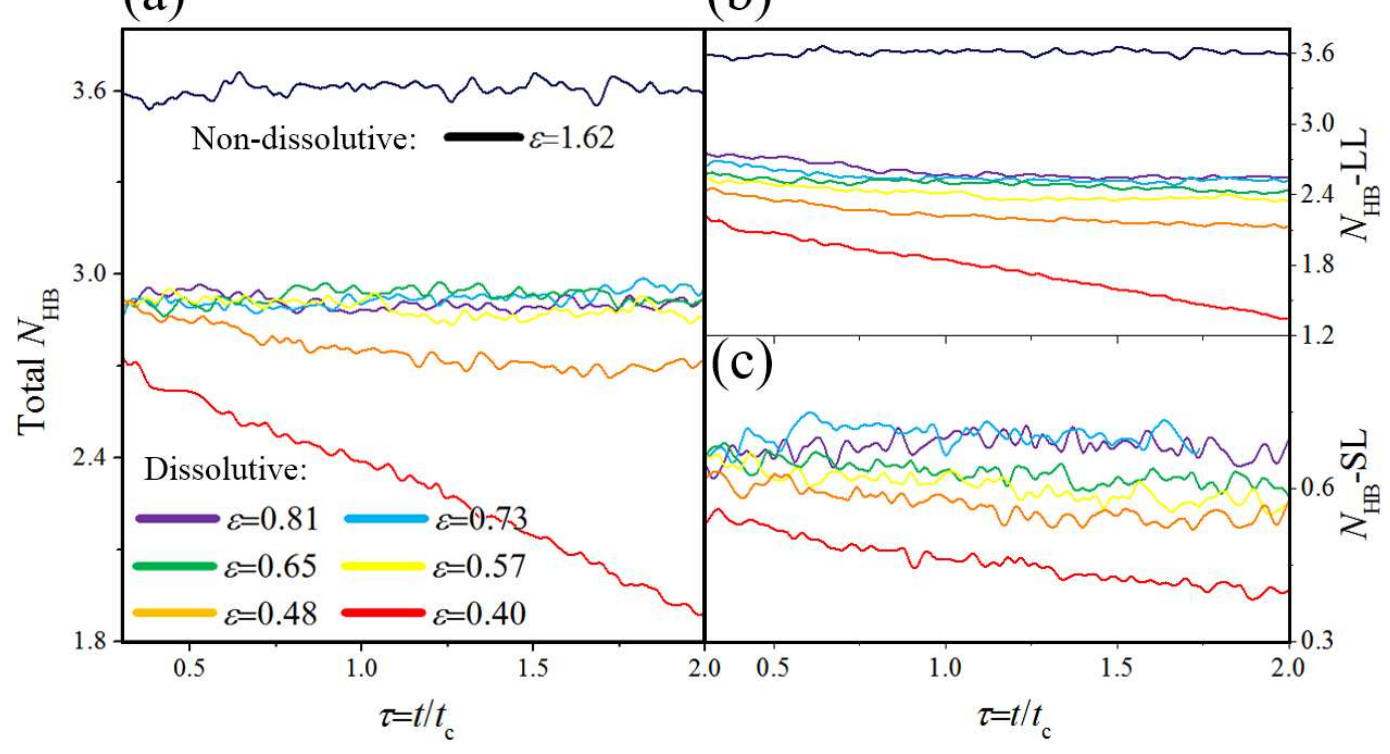

Figure 8. Evolution of the average number of hydrogen bonds (a) in the droplet, (b) formed between water molecules, and (c) formed between dissolved solid particles and water molecules.

Network of hydrogen bonds (HBs). HBs makes liquid water exceptional. Geometric criterion were used to judge whether hydrogen bond formed. ${ }^{43}$ We specified a hydrogen bond between a water molecule and other molecule if the O-P distance was less than $3.5 \AA$, the angle O-H $\cdots \mathrm{P}$ was greater than $120^{\circ}$, and the $\mathrm{H}^{\cdots} \mathrm{P}$ distance was less than $2.5 \AA$, where $\mathrm{O}$ and $\mathrm{H}$ are oxygen and hydrogen atoms in one water molecule. P could be an oxygen atom in another water molecule or a dissolved solid particle. In non-dissolutive wetting, the average number of $\mathrm{HB}\left(N_{\mathrm{HB}}\right)$ of a water molecule remains about 3.6 (black lines in Figure 8), which is in good agreement with the literature. ${ }^{44}$ In dissolutive wettings, the average number $N_{\mathrm{HB}}$ between water molecules decreases with the decrease of $\varepsilon$. When $\varepsilon$ is large, $N_{\mathrm{HB}}$ between water molecules decreases to about 2.5 (Figure 8b), while there are HBs between the dissolved particles and the water molecules (Figure 8c). Hence, the total $N_{\mathrm{HB}}$ is about 2.9 for solid with low dissolubility. When $\varepsilon$ is small, $N_{\mathrm{HB}}$ 
between water molecules greatly decreases, while $N_{\mathrm{HB}}$ between water molecule and dissolved particles also decreases. Therefore, the total $N_{\mathrm{HB}}$ significantly decreases for solid with high dissolubility.

\section{CONCLUSIONS}

In conclusion, the advancing of liquid on dissolvable solids depended on both the interface and the dissolving energies. The droplets spread on an evolving solid surface following a different dynamic law, compared with spreading on non-dissolutive surfaces. The scaling exponent $\alpha$ varies from $1 / 10$ to $1 / 7$ in dissolutive wetting, while $\alpha$ is $1 / 7$ in non-dissolutive wetting. Contact line interacts with the receding solid surface through the hydration shells, pulling the solid particles to dissolve, varying the liquid/interface properties and changing the flow field in the droplet. Reflected by PDF analysis, the atomic structure of the liquid changes with the increasing dissolved particles. Consequently, the hydrogen bond is influenced by the coupled mass and momentum transport. Our findings may help to understand the dynamics of dissolutive wetting and assist future design in practical applications.

\section{ACKNOWLEDGEMENT}

This work was jointly supported by the National Natural Science Foundation of China (NSFC, Grant Nos. 11672300, 11611130166 and U1562105), Royal Society-Newton Mobility Grant (Ref. IE150674), CAS Key Research Program of Frontier Sciences (Grant No. QYZDJ-SSWJSC019) and the Strategic Priority Research Program of the Chinese Academy of Sciences (Grant No. XDB22040401). 


\section{REFERENCES}

(1) Lasaga, A. C.; Luttge, A. Variation of Crystal Dissolution Rate Based on a Dissolution Stepwave Model. Science 2001, 291 (5512), 2400-2404.

(2) Colombani, J. The Alkaline Dissolution Rate of Calcite. J. Phys. Chem. Lett. 2016, 7 (13), 2376-2380.

(3) Webb, E. B.; Grest, G. S.; Heine, D. R. Precursor film controlled wetting of Pb on $\mathrm{Cu}$. Phys. Rev. Lett. 2003, 91 (23), 236102.

(4) Thiele, U.; John, K.; Bär, M. Dynamical model for chemically driven running droplets. Phys. Rev. Lett. 2004, 93 (2), 027802.

(5) Villanueva, W.; Boettinger, W. J.; McFadden, G. B.; Warren, J. A. A diffuse-interface model of reactive wetting with intermetallic formation. Acta Mater. 2012, 60 (9), 37993814.

(6) Ristroph, L.; Moore, M. N.; Childress, S.; Shelley, M. J.; Zhang, J. Sculpting of an erodible body by flowing water. Proc. Nat. Acad. Sci. USA 2012, 109 (48), 19606-19609.

(7) Foreman, B. Z.; Lai, S. Y.; Komatsu, Y.; Paola, C. Braiding of submarine channels controlled by aspect ratio similar to rivers. Nat. Geosci. 2015, 8 (9), 700-703.

(8) Cermeño, P.; Falkowski, P. G.; Romero, O. E.; Schaller, M. F.; Vallina, S. M. Continental erosion and the Cenozoic rise of marine diatoms. Proc. Nat. Acad. Sci. USA 2015, 112 (14), 4239-4244.

(9) Dokoumetzidis, A.; Macheras, P. A century of dissolution research: from Noyes and Whitney to the biopharmaceutics classification system. Int. J. Pharm. 2006, 321 (1), 1-11.

(10) Siepmann, J.; Peppas, N. Modeling of drug release from delivery systems based on hydroxypropyl methylcellulose (HPMC). Adv. Drug Deliver. Rev. 2012, 64, 163-174.

(11) Schneider, J.; Reuter, K. Efficient Calculation of Microscopic Dissolution Rate Constants: The Aspirin-Water Interface. J. Phys. Chem. Lett. 2014, 5 (21), 3859-3862.

(12) Bain, C. D.; Whitesides, G. M. A study by contact angle of the acid-base behavior of monolayers containing. omega.-mercaptocarboxylic acids adsorbed on gold: an example of reactive spreading. Langmuir 1989, 5 (6), 1370-1378.

(13) Klajn, R. Spiropyran-based dynamic materials. Chem. Soc. Rev. 2014, 43 (1), 148-184.

(14) Bain, C. D.; Burnett-Hall, G. D.; Montgomerie, R. R. Rapid motion of liquid drops. Nature 1994, 372 (6505), 414-415. 
(15) Domingues Dos Santos, F.; Ondarcuhu, T. Free-running droplets. Phys. Rev. Lett. 1995, 75 (16), 2972.

(16) Bico, J.; Quéré, D. Self-propelling slugs. J. Fluid. Mech. 2002, 467, 101-127.

(17) Daccord, G.; Lenormand, R. Fractal patterns from chemical dissolution. Nature 1987, 325 (6099), 41-43.

(18) Dupas, J.; Verneuil, E.; Ramaioli, M.; Forny, L.; Talini, L.; Lequeux, F. Dynamic wetting on a thin film of soluble polymer: effects of nonlinearities in the sorption isotherm. Langmuir 2013, 29 (40), 12572-12578.

(19) De Gennes, P.-G.; Brochard-Wyart, F.; Quéré, D. Capillarity and wetting phenomena: drops, bubbles, pearls, waves; Springer: New York, 2004.

(20) Su, S.; Yin, L.; Sun, Y.; Murray, B. T.; Singler, T. J. Modeling dissolution and spreading of Bi-Sn alloy drops on a Bi substrate. Acta Mater. 2009, 57 (10), 3110-3122.

(21) Huang, J. M.; Moore, M. N. J.; Ristroph, L. Shape dynamics and scaling laws for a body dissolving in fluid flow. J. Fluid. Mech. 2015, 765, R3.

(22) Sun, W.; Yang, F. Q. Evaporation-induced formation of self-organized gradient concentric rings on sub-micron pre-cast PMMA films. Soft Matt. 2014, 10 (25), 4451-4457.

(23) Sun, W.; Yang, F. Q. Fabrication of asymmetric-gradient-concentric ring patterns via evaporation of droplets of PMMA solution at different substrate temperatures. RSC Adv. 2015, 5 (38), 29850-29858.

(24) Bonn, D.; Eggers, J.; Indekeu, J.; Meunier, J.; Rolley, E. Wetting and spreading. Rev. Mod. Phys. 2009, 81 (2), 739-805.

(25) De Gennes, P.-G. Wetting: statics and dynamics. Rev. Mod. Phys. 1985, 57 (3), 827-863.

(26) Yuan, Q. Z.; Zhao, Y. P. Precursor film in dynamic wetting, electrowetting, and electroelasto-capillarity. Phys. Rev. Lett. 2010, 104 (24), 246101.

(27) Sui, Y.; Ding, H.; Spelt, P. D. Numerical simulations of flows with moving contact lines. Annu. Rev. Fluid. Mech. 2014, 46, 97-119.

(28) Extrand, C. W. Origins of Wetting. Langmuir 2016, 32 (31), 7697-7706.

(29) Tanner, L. The spreading of silicone oil drops on horizontal surfaces. J. Phys. D: Appl. Phys. 1979, 12, 1473-1484.

(30) Yuan, Q. Z.; Zhao, Y. P. Multiscale dynamic wetting of a droplet on a lyophilic pillararrayed surface. J. Fluid. Mech. 2013, 716, 171-188. 
(31) Warren, J. A.; Boettinger, W.; Roosen, A. Modeling reactive wetting. Acta Mater. 1998, $46(9), 3247-3264$.

(32) Plimpton, S. Fast parallel algorithms for short-range molecular-dynamics. J. Comput. Phys. 1995, 117 (1), 1-19.

(33) González, M. A.; Abascal, J. L. F. The shear viscosity of rigid water models. J. Chem. Phys. 2010, 132 (9), 096101.

(34) Vega, C.; De Miguel, E. Surface tension of the most popular models of water by using the test-area simulation method. J. Chem. Phys. 2007, 126 (15), 154707.

(35) Noyes, A. A.; Whitney, W. R. The rate of solution of solid substances in their own solutions. J. Am. Chem. Soc. 1897, 19 (12), 930-934.

(36) De Gennes, P.-G. The dynamics of reactive wetting on solid surfaces. Physica. A 1998, 249 (1), 196-205.

(37) Savitzky, A.; Golay, M. J. Smoothing and differentiation of data by simplified least squares procedures. Anal. Chem. 1964, 36 (8), 1627-1639.

(38) Glasstone, S.; Laidler, K.; Eyring, H. The Theory of Rate Processes; McGraw-Hill: New York, 1941.

(39) Blake, T. D.; de Coninck, J. The influence of solid-liquid interactions on dynamic wetting. Adv. Colloid Interf. Sci. 2002, 96 (1-3), 21-36.

(40) Webb, E. B.; Grest, G. S. Liquid/vapor surface tension of metals: Embedded atom method with charge gradient corrections. Phys. Rev. Lett. 2001, 86 (10), 2066.

(41) Bahadur, R.; Russell, L. M.; Alavi, S. Surface tensions in NaCl-water-air systems from MD simulations. J. Phys. Chem. B 2007, 111 (41), 11989-11996.

(42) Zhong, J.; Zhao, Y.; Li, L.; Li, H.; Francisco, J. S.; Zeng, X. C. Interaction of the NH2 Radical with the Surface of a Water Droplet. J. Am. Chem. Soc. 2015, 137 (37), 1207012078.

(43) Martins Costa, M. T. C. QM/MM simulations of polyols in aqueous solution. J. Mol. Struc. - THEOCHEM 2005, 729 (1-2), 47-52.

(44) Rastogi, A.; Ghosh, A. K.; Suresh, S. Hydrogen Bond Interactions Between Water Molecules in Bulk Liquid, Near Electrode Surfaces and Around Ions. In Thermodynamics - Physical Chemistry of Aqueous Systems; INTECH, 2011, pp 351-364. 
TOC Graphic

Dynamic wetting of a liquid droplet on a dissolvable substrate.

\section{Dissolutive wetting}

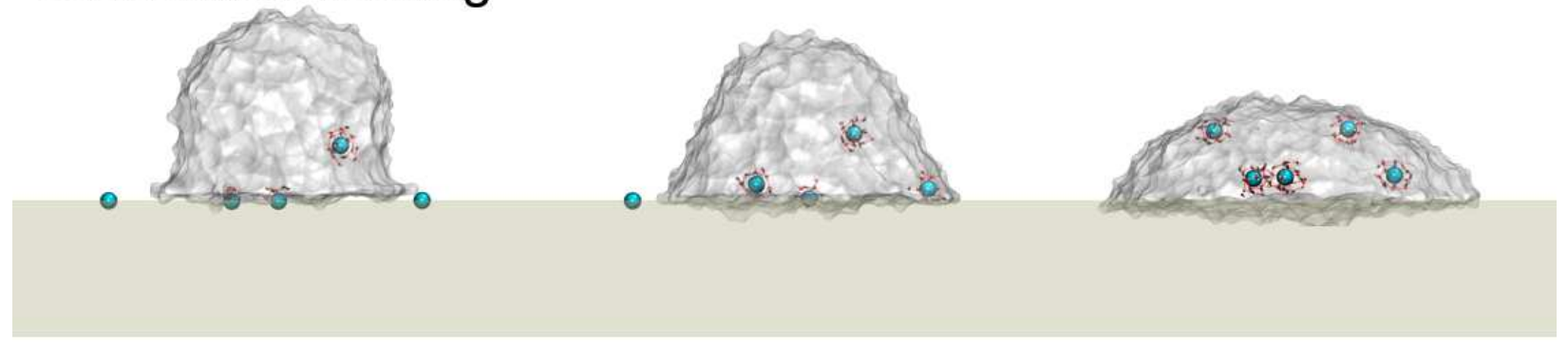

Flow field

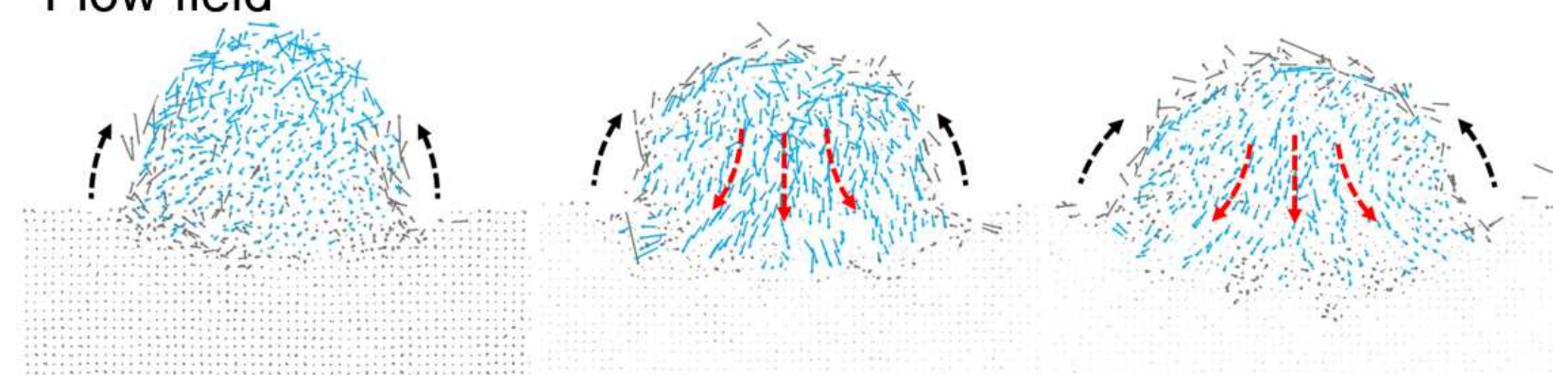

